Check for updates

Cite this: Chem. Sci., 2019, 10, 7779

๑ All publication charges for this article have been paid for by the Royal Society of Chemistry

Received 25th April 2019

Accepted 2nd July 2019

DOI: 10.1039/c9sc02039j

rsc.li/chemical-science

\section{Structure and effective charge characterization of proteins by a mobility capillary electrophoresis based method}

\author{
Wenjing Zhang, (D) ${ }^{a}$ Haimei Wu, ${ }^{a}$ Rongkai Zhang, ${ }^{a}$ Xiang Fang ${ }^{b}$ and Wei Xu (D) *a
}

Measuring the conformations and effective charges of proteins in solution is critical for investigating protein bioactivity, but their rapid analysis remains a challenging problem. Here we report a mobility capillary electrophoresis (MCE) based method for the rapid analysis of protein stereo-structures and effective charges in different solution environments. With the capability of mixture separation, MCE measures the hydrodynamic radius of a protein through Taylor dispersion analysis and its effective charge through ion mobility analysis. The experimental results acquired from MCE are then utilized to restrain molecular dynamics simulations, so that the most probable conformation of that protein can be obtained. As proof-of-concept demonstrations, the charge states and structures of five proteins were analyzed under close to native environments. The conformation transitions and charge state variations of bovine serum albumin and lysozyme under different $\mathrm{pH}$ conditions were also investigated. This method is promising for high-throughput protein analysis, which could potentially be coupled with mass spectrometry for investigating protein stereo-structures and functions in top-down proteomics.

\section{Introduction}

Proteins perform a vast number of biological functions in living organisms. ${ }^{1,2}$ Besides its primary sequence, the function of a protein greatly depends on its stereo-structure, ${ }^{3-5}$ as well as its effective charge in solution., ${ }^{6,7}$ The protein conformation and effective charge could also influence each other. To understand the working mechanism of a biosystem or the physiological functions of proteins, ${ }^{8,9}$ it is essential to identify not only the expression level of proteins, but also their structures. Highresolution protein structure analytical techniques, such as X-ray diffraction, ${ }^{10}$ nuclear magnetic resonance (NMR) ${ }^{11}$ and electron microscopy, ${ }^{12}$ are available. To obtain a high-resolution protein structure, high abundance samples are typically required and the whole process is time-consuming, which limits these techniques from use in large-scale protein analysis. ${ }^{\mathbf{1 3 , 1 4}}$ On the other hand, large-scale protein analysis has been carried out in proteomics, in which the entire set of proteins in an organism or biosystem are studied typically through the combination of liquid chromatography (LC) or capillary electrophoresis (CE) with mass spectrometry (MS). ${ }^{15-17}$ Protein primary sequences and posttranslational modifications (PTMs) could be resolved through tandem MS. ${ }^{18}$ However, protein stereo-structure information, ${ }^{19}$ as well as its effective charge in solution, are mostly missing. As

${ }^{a}$ School of Life Science, Beijing Institute of Technology, No. 5 South Zhongguancun Street, Haidian Dist, Beijing, China.E-mail: weixu@bit.edu.cn

${ }^{b}$ National Institute of Metrology, No. 18, Bei San Huan Dong Lu, Chaoyang Dist, Beijing, China a result, biological phenotypic variances caused by protein stereostructural differences would be masked.

To distinguish and separate molecular isoforms, ion mobility spectrometry (IMS) is increasingly integrated into MS instruments. $^{20}$ Collision cross sections (CCSs) of ions ${ }^{21}$ after gasification and ionization could be obtained. Ion $\mathrm{CCSs}^{22}$ could reflect ion structures in the gas phase, ${ }^{23,24}$ which may not have a direct relationship with their corresponding structures in solution. ${ }^{25}$ Protein charge states in the gas phase ${ }^{26}$ also have no correlation with its effective charge in solution. ${ }^{7}$ Taylor dispersion analysis (TDA) is a liquid-phase structural analysis technique, through which the diffusion coefficient and hydrodynamic radius of a particle or molecule can be measured. ${ }^{27}$ In TDA experiments, a constant pressure was applied to drive analytes in a laminar flow, which could easily be realized on a CE instrument. ${ }^{28}$ Since electroosmotic flow (EOF) in a CE experiment would destroy the laminar flow field distribution, ${ }^{29}$ no electric field was applied in conventional TDA methods. Therefore, conventional TDA methods do not have mixture separation/analysis capabilities. $^{30}$ To address this problem, a time-sharing technique was proposed by Billy A. Williams and Gyula Vigh, in which analytes were first separated by operating a CE instrument in a conventional CE mode and then structure analyzed by tuning the instrument into the TDA mode. With this two-step operation, the hydrodynamic radiuses of different molecules in a mixture could be measured. ${ }^{31}$

Recently, mobility capillary electrophoresis (MCE) was proposed to achieve ion separation and hydrodynamic radius measurement simultaneously. ${ }^{32,33}$ In MCE experiments, ions 
were driven in a constant flow ${ }^{34,35}$ and separated by an electric field, making MCE a liquid-phase analogue of IMS. With minimized $\mathrm{EOF}^{36-38}$ ion electrophoresis migration could be precisely determined, and the ion hydrodynamic radius was calculated from ion migration time. After the spheroidal approximation, the ion hydrodynamic radius of a protein obtained from MCE defines a mathematical relationship between the length and diameter of this spheroid, which could then be applied to select the most probable protein conformation in solution from molecular dynamics (MD) simulation results. However, in previous studies, the ion effective charge state needed to be estimated from theoretical analysis, which limited its application in terms of both solution and analyte categories. Specifically, only proteins in a solution with no or very low salt concentrations can be analyzed. However, salts are present in many practical biosystems, and their presence may help in maintaining the native conformations of proteins. Furthermore, the accuracy of protein charge state estimation also limits the accuracy of ion hydrodynamic radius calculation. ${ }^{32,33} \mathrm{In}$ fact, the protein charge state is an important factor affecting its activity and solubility, ${ }^{39}$ and it may affect the protein conformation in turn. ${ }^{40-42}$ Its measurement in solution is a challenging problem in itself. ${ }^{43}$ Through chemical modifications, the protein charge ladder method has been developed to study the effective charge of each protein. ${ }^{6,44}$ The capillary isotachophoresis (ITP) method was also developed to determine the effective charges of polymers and polycationic peptides. ${ }^{45-47}$ In summary, although the established structural biology technologies could provide high resolution protein structure information, they have relatively low throughput. High throughput proteomic methods could provide little protein stereo-structure information. ${ }^{48}$ Additional methods are essential for bridging this gap.

To overcome these limitations, a new MCE based method was proposed in this work. As the first attempt to integrate TDA into MCE experiments, this method allows ion separation, hydrodynamic radius and effective charge measurements in different solution environments to be achieved in a single experiment. Without the need for any chemical modification, ion dispersion analysis was first performed on the shape of a peak to obtain the ion hydrodynamic radius; ion mobility analysis was then carried out on peak retention time to acquire the ion effective charge. As proof-of-concept demonstrations, peptide and protein mixtures were studied. The ion hydrodynamic radiuses obtained using this method were first confirmed with conventional TDA results. The isoelectric point of a protein estimated using this MCE based method also agrees with that in the literature. Next, by coupling with MD simulations, the native conformations ${ }^{49}$ of three proteins were first analyzed with results confirmed by protein data bank (PDB) results. Unfolding and structural variations of two other proteins induced by $\mathrm{pH}$ changes were also explored. ${ }^{50}$

\section{Theory}

In an MCE experiment as shown in Fig. 1, ions in a constant liquid flow will experience an electric field force. ${ }^{32}$ The constant

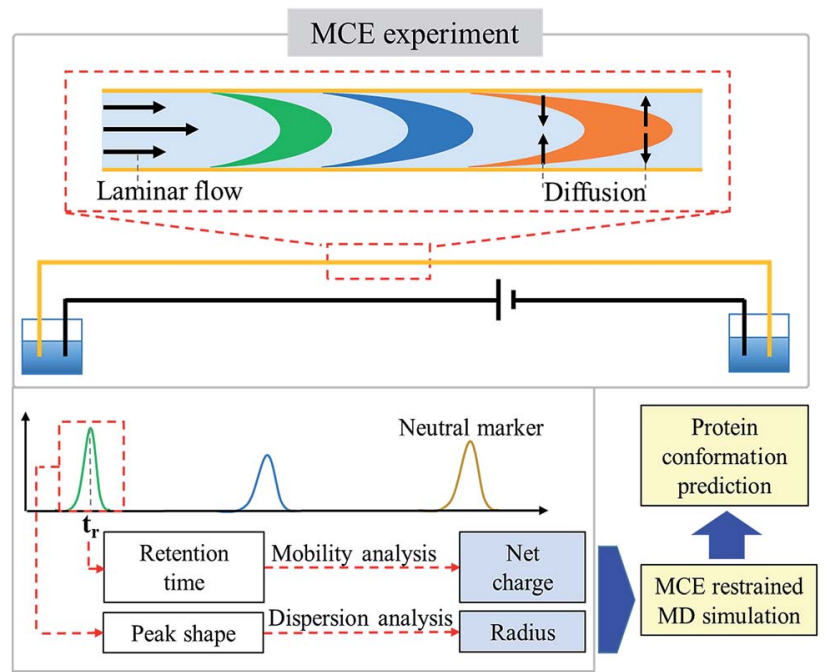

Fig. 1 Schematic diagram of the MCE based method for protein conformation and charge state analyses. Driven by the laminar flow, ions were separated by the electric field in MCE experiments. The ion hydrodynamic radius and effective charge were obtained by performing dispersion analysis on peak shape and ion mobility analysis on retention time, respectively. Applying MCE results as a selection rule for MD simulations, the most probable conformations of proteins in solution were then acquired.

liquid flow is maintained by applying a constant pressure over the separation channel. Coated silica capillaries were used as the separation channel, so that electroosmotic flow (EOF) was minimized and not considered. ${ }^{38}$ As a result, a stable laminar flow is formed within the separation channel, which is the same as that in a TDA experiment. ${ }^{51,52}$ Different from a TDA experiment, a separation voltage was also applied during an MCE experiment. In the presence of both the electric field and the laminar flow field, analyte ions could be separated based on their charge states and hydrodynamic radiuses. ${ }^{53-55}$ To accurately measure the hydrodynamic radius and effective charge of an ion, ${ }^{56}$ four steps were carried out, including ion velocity distribution analysis, diffusion coefficient calculation, temperature and viscosity correction, and ion effective charge calculation, in which the $\mathrm{TDA}^{57-59}$ and electrokinetic dispersion (EKD) theories ${ }^{54,55}$ were applied.

\section{Ion velocity distribution analysis}

By applying a constant pressure, a laminar flow is expected within the silica capillary. The velocity profile $(v(r))$ has a parabolic shape over the cross section of the capillary. ${ }^{60,61}$

$$
v(r)=v_{\mathrm{m}}\left(1-r^{2} / R_{\mathrm{c}}^{2}\right)
$$

where $v_{\mathrm{m}}$ is the maximum fluid velocity at the center of the capillary, $r$ is the radial distance from the capillary center and $R_{\mathrm{c}}$ is the capillary inner radius. In the presence of a separation electric field, the velocity of an ion $(u(r))$ would be different from that of the liquid flow. When a constant DC voltage is applied, ${ }^{55}$

$$
u(r)=v(r)+q U /(L \xi)
$$


where $q$ is the effective charge that the ion possesses, $U$ is the DC voltage applied across the capillary with a length $L$, and $\xi=$ $6 \pi \eta R_{\mathrm{h}}$, where $\eta$ is the viscosity coefficient of the buffer solution and $R_{\mathrm{h}}$ is the hydrodynamic radius of the ion.

The second term in eqn (2) is a constant for the same ion species. Eqn (2) shows that different ion species would have different velocity profiles, and thus they could be separated. Furthermore, for each type of ion, its velocity distribution in MCE experiments also has a parabolic shape, which is the same as the velocity profile in TDA experiments. Therefore, ion dispersion in the radial direction in an MCE experiment is also the same as that in a TDA experiment.

\section{Diffusion coefficient calculation in MCE}

With the same ion velocity profile, similar derivation procedures as in TDA could be applied in the dispersion coefficient calculation in MCE experiments. Developed by Westhaver, ${ }^{62,63}$ Taylor, ${ }^{64}$ and Aris, ${ }^{65}$ the TDA approach was further studied by Datta and Kotamarthi (1990) to obtain the following electrokinetic dispersion coefficient $\left(K_{\mathrm{i}}\right)$ expression $^{54,55}$ for the general case involving both pressure driven and electroosmotic flows:

$$
K_{\mathrm{i}}=D+R_{\mathrm{c}}^{2}\left(v_{\mathrm{p}}^{2}+\partial_{2} v_{\mathrm{p}} v_{\mathrm{eo}}+\partial_{3} v_{\mathrm{eo}}^{2}\right) /(48 D)
$$

where $D$ is the diffusion coefficient, $v_{\mathrm{p}}$ and $v_{\text {eo }}$ are the areaaveraged Poiseuille and electroosmotic flow velocities, respectively, and $\partial_{2}$ and $\partial_{3}$ are dimensionless constants related to the dimensionless capillary radius. When $v_{\text {eo }}=0$, eqn (3) reduces to the "Taylor-Aris" expression. Since the EOF in MCE is also minimized and not considered, ${ }^{38}$ eqn (3) will reduce to

$$
K_{\mathrm{i}}=D+R_{\mathrm{c}}{ }^{2} v_{\mathrm{p}}{ }^{2} /(48 D)
$$

Under the same assumption as in TDA that the ion dispersion along the capillary axis is negligible, which is $D \ll R_{\mathrm{c}}{ }^{2} v_{\mathrm{p}}{ }^{2} /$ $(48 D),{ }^{57}$

$$
K_{\mathrm{i}}=R_{\mathrm{c}}{ }^{2} v_{\mathrm{p}}{ }^{2} /(48 D)
$$

$v_{\mathrm{p}}$ could be measured by adding a neutral marker into the system, and $v_{\mathrm{p}}=L_{\mathrm{t}} / t_{\mathrm{p}}$, where $L_{\mathrm{t}}$ is the length of the capillary from the injection point to the ultraviolet (UV) detection window and $t_{\mathrm{p}}$ is the arrival time (or retention time) of the neutral marker.

Utilizing the fact that the ion dispersion in the radial direction in an MCE experiment is the same as that in a TDA experiment, the recorded peak should have a Gaussian distribution. The peak arrival time (or retention time, $t_{\mathrm{r}}$ ) and temporal standard deviation $(\sigma)$ of the peak ${ }^{55}$ could be used to calculate its electrokinetic dispersion coefficient $\left(K_{\mathrm{i}}\right)$ :

$$
\sigma^{2}=2 K_{\mathrm{i}} t_{\mathrm{r}} / u^{2}
$$

where $u$ is the averaged migration velocity of one species of ions, and it could be measured as $u=L_{\mathrm{t}} / t_{\mathrm{r}}$. Combining eqn (5) and (6), we will have

$$
D=\frac{R_{\mathrm{c}}{ }^{2} t_{\mathrm{r}} v_{\mathrm{p}}{ }^{2}}{24 \sigma^{2} u^{2}}=\frac{R_{\mathrm{c}}{ }^{2} t_{\mathrm{r}}{ }^{3}}{24 \sigma^{2} t_{\mathrm{p}}{ }^{2}}
$$

As shown in eqn (7), the ion diffusion coefficient could be obtained by measuring its peak retention time and temporal standard deviation of the peak, but it also requires introducing a neutral marker and measuring its retention time. Subsequently, the protein hydrodynamic radius $\left(R_{\mathrm{h}}\right)$ could be calculated from the Stokes equation:

$$
R_{\mathrm{h}}=k_{\mathrm{B}} T /(6 \pi \eta D)
$$

where $k_{\mathrm{B}}$ is the Boltzmann constant, and $T$ is the temperature in the capillary. It should be noted that the concentration ratio of sample to background electrolyte (BGE) should be adjusted, so that the electro-migration dispersion effect is minimized. ${ }^{66} \mathrm{~A}$ strong electro-migration dispersion effect would cause the peak tailing effect, which results in distorted and unsymmetric peaks. ${ }^{67}$ It is found that this electro-migration dispersion could be minimized as long as the concentration of BGE is much larger than that of the sample. In turn, the concentration of the BGE could be reduced by lowering the sample concentrations, which would require the use of more sensitive CE detectors, such as fluorescence detectors.

\section{Temperature and viscosity correction}

With the introduction of a separation voltage into the MCE system, the effects of thermal heating ${ }^{\mathbf{6 8 , 6 9}}$ need to be considered and corrected for before we can accurately calculate the ion hydrodynamic radius. Although most commercial CE instruments have temperature control, the heat generated inside the capillary may not be conducted instantaneously, and a higher temperature may be present inside the capillary. ${ }^{70}$ In turn, temperature also affects the viscosity of the solvent, which is another important parameter when calculating the ion hydrodynamic radius. Since a neutral marker is present in the system in every MCE run, temperature over viscosity in every MCE run was corrected for as

$$
\frac{T}{\eta}=\frac{6 \pi R_{\mathrm{hn}} D_{\mathrm{n}}}{k_{\mathrm{B}}}
$$

where $R_{\mathrm{hn}}$ is the known hydrodynamic radius of a neutral marker (for instance, the hydrodynamic radius of phenol used in this study is $0.27 \mathrm{~nm}$ obtained by the TDA method ${ }^{71}$ ), and $D_{\mathrm{n}}$ could be obtained using eqn (7). After correction, eqn (8) becomes

$$
R_{\mathrm{h}}=\frac{R_{\mathrm{hn}} D_{\mathrm{n}}}{D}
$$

It should be noticed that the average thermal effect was corrected for using the neutral marker in this study. Spatially varying temperature fields were not considered.

\section{Ion effective charge calculation}

After determining the hydrodynamic radius of an ion, the effective charge of that ion could then be obtained by 
performing mobility analysis of ion retention time $\left(t_{\mathrm{r}}\right)$ as shown in Fig. 1. Ion retention time is determined from the ion effective charge $(q)$ and the ion hydrodynamic radius,

$$
L_{\mathrm{t}}=\left(v_{\mathrm{p}}+q U /(L \xi)\right) t_{\mathrm{r}}
$$

Thus

$$
q=n e=\frac{6 \pi \eta R_{\mathrm{h}} L}{U}\left(\frac{L_{\mathrm{t}}}{t_{\mathrm{r}}}-\frac{L_{\mathrm{t}}}{t_{\mathrm{p}}}\right)
$$

where $n$ is the charge number and $e$ is the elementary charge value.

\section{MCE restrained protein structure modelling}

As demonstrated in previous publications, ${ }^{32,33}$ MCE based experimental results could be used as a selection rule for MD simulations to predict the most probable protein structures in solution. However, the previous method requires the estimation of ion charge states before the ion hydrodynamic radius could be obtained. Charge states of proteins were estimated through $\mathrm{p} K_{\mathrm{a}}$ calculations. Theoretical charge state calculation was heavily limited to solvents with no salt or very low salt concentrations. Nevertheless, an appropriate salt concentration is essential in terms of maintaining the native structures of biomolecules. The method proposed in this study could measure both the hydrodynamic radius and effective charge of a biomolecule, and it could work under different salt and $\mathrm{pH}$ conditions. After obtaining the ion hydrodynamic radius, similar procedures could be carried out, including spheroid approximation and matching with MD simulations, so that the most probable protein conformations under different solvent conditions could be obtained.

\section{Experimental section}

\section{Chemicals}

Peptides bradykinin and VEALYL (pentapeptide) were synthesized by SBS Genetech Co. Ltd. (Beijing, China). All proteins including insulin, cytochrome $\mathrm{C}$, ribonuclease A (RNase A), lysozyme and bovine serum albumin (BSA) were purchased from Sigma Aldrich (St. Louis, MO, USA). Sodium dihydrogen phosphate dihydrate $\left(\mathrm{NaH}_{2} \mathrm{PO}_{4} \cdot 2 \mathrm{H}_{2} \mathrm{O}\right)$, di-sodium hydrogen phosphate $\left(\mathrm{Na}_{2} \mathrm{HPO}_{4} \cdot 12 \mathrm{H}_{2} \mathrm{O}\right)$, sodium chloride $(\mathrm{NaCl})$, citric acid monohydrate $\left(\mathrm{C}_{6} \mathrm{H}_{8} \mathrm{O}_{7} \cdot \mathrm{H}_{2} \mathrm{O}\right)$ and phenol were purchased from Aldrich Chemical Co. (Milwaukee, WI). Pure water used in this work was purchased from Wahaha company (Hangzhou, China).

The above chemicals were used for the preparation of sample and buffers. Phosphate buffer with $\mathrm{pH} 7$ was prepared by mixing $6.1 \mathrm{~mL}$ of $0.2 \mathrm{~mol} \mathrm{~L}^{-1} \mathrm{Na}_{2} \mathrm{HPO}_{4}$ and $3.9 \mathrm{~mL}$ of $0.2 \mathrm{~mol} \mathrm{~L}^{-1}$ $\mathrm{NaH}_{2} \mathrm{PO}_{4}$. Disodium hydrogen-phosphate citric acid buffers were also prepared to set the $\mathrm{pH}$ values at 2.2, 3.4, 5, 6 and 7 . The disodium hydrogen-phosphate citric acid buffers with $\mathrm{pH}$ $2.2,3.4,5,6$, and 7 were prepared by the mixing of $0.40 \mathrm{~mL}, 5.70$ $\mathrm{mL}, 10.30 \mathrm{~mL}, 12.63 \mathrm{~mL}$ and $16.47 \mathrm{~mL}$ of $0.2 \mathrm{~mol} \mathrm{~L}^{-1} \mathrm{Na}_{2} \mathrm{HPO}_{4}$ with $10.60 \mathrm{~mL}, 14.30 \mathrm{~mL}, 9.70 \mathrm{~mL}, 7.37 \mathrm{~mL}$ and $3.53 \mathrm{~mL}$ of $0.1 \mathrm{~mol} \mathrm{~L}^{-1} \mathrm{C}_{6} \mathrm{H}_{8} \mathrm{O}_{7}$, respectively. These buffers were diluted 10 times to dissolve peptides and proteins to their final concentrations. Phenol was selected as the neutral marker to calculate the retention time driven by pressure, and it was also used to correct for temperature and viscosity variations. 1/1000 (volume ratio) phenol was added in every sample solution, unless otherwise specified.

\section{MCE experiment}

A commercial Lumex CE system equipped with a UV detector (model Capel 105M, St. Petersburg, Russia) was used to perform MCE experiments. All capillaries used in the MCE experiment were coated capillaries obtained from Sino Suntech (Hebei, China), which have stable neutral polymer coatings (poly $\mathrm{N}$ isopropyl-acrylamide, PAM). Capillaries with $75 \mu \mathrm{m}$ id, $60 \mathrm{~cm}$ total length, and $50 \mathrm{~cm}$ effective length were used. Before each working day, the capillary should be rinsed with water for $10 \mathrm{~min}$, and then rinsed with the buffer solution for $10 \mathrm{~min}$. The capillary was flushed with the buffer for 5 min between all of the runs. Sample injection was performed using a pressure of 50 mbar for $5 \mathrm{~s}$. Most experiments in this paper use $50 \mathrm{mbar}$ to maintain laminar flow in the separation channel and $10 \mathrm{kV}$ to separate ions, unless otherwise specified. The UV detector works at a wavelength of $214 \mathrm{~nm} .25{ }^{\circ} \mathrm{C}$ was set for the capillary during experiments.

\section{MD simulation}

MD simulations were performed using the GROMOS54A7 force field ${ }^{72}$ in GROMACS 2016.1. ${ }^{73}$ The initial structure of a protein was obtained from the RCSB Protein Data Bank (PDB ID: 1APH; 1AKK; 1RUN; 4F5S; 1GXV). $\mathrm{p} K_{\mathrm{a}}$ calculations were performed with PROPKA. ${ }^{74,75}$ The systems were placed in a cubic box, solvated with explicit simple point charge (SPC) model water molecules. ${ }^{76}$ The counter ions (chloride at $\mathrm{pH} 7$, formate at $\mathrm{pH}$ 3) were added as necessary to ensure system neutrality. The model was then energetically stabilized by the steepest descent algorithm, followed by an equilibration for at least $1 \mathrm{~ns}$ in water at $300 \mathrm{~K}$. Periodic boundary conditions were used with a $1.4 \mathrm{~nm}$ cut off for non-bonded interactions. Long-range electrostatic corrections were taken into account by the particle mesh Ewald method. ${ }^{77}$ The overall simulation window was $100 \mathrm{~ns}$. The rootmean-square deviation (RMSD) of the radius of gyration was monitored throughout all simulations.

\section{Results and discussion}

\section{Method validation}

First, MCE experiments were carried out for a peptide (bradykinin, $0.24 \mu \mathrm{mol} \mathrm{mL}^{-1}$ ) by applying different separation voltages $(5,10$ and $20 \mathrm{kV})$. The phosphate buffer was used as the running buffer (20 mM, pH 7). After sample injection (50 mbar, $5 \mathrm{~s}$ ), a pressure of 50 mbar was then applied to generate the constant liquid flow during MCE separation processes. Phenol $(1 / 4000$, volume ratio) was used as the neutral marker. As shown in Fig. 2a, the bradykinin peak would separate further away from the phenol peak with increased separation voltages. Retention time variation also exists for phenol peaks among 

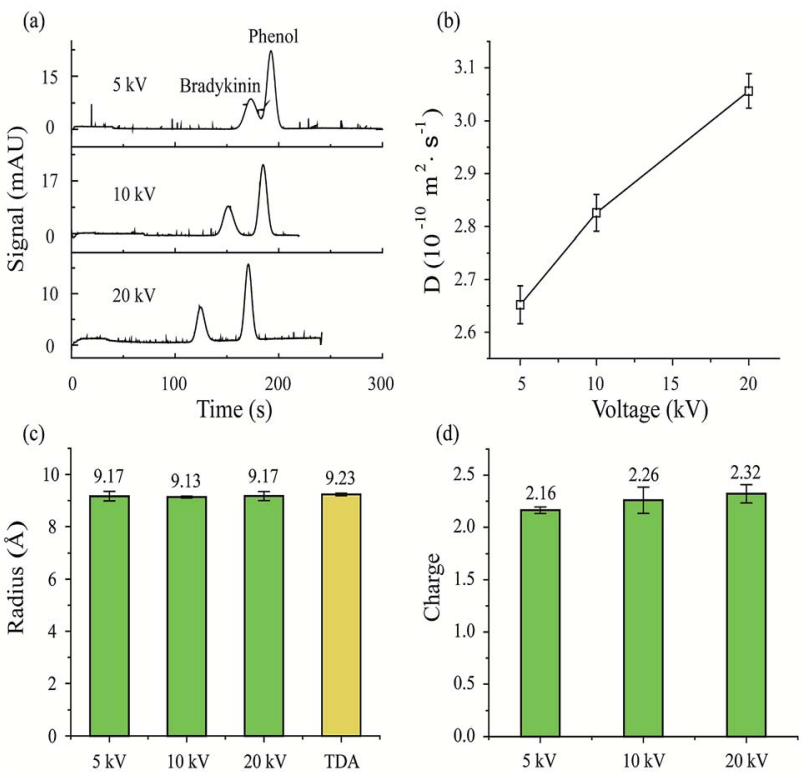

Fig. 2 Structure and effective charge measurements of bradykinin under different MCE conditions. (a) Electropherograms of bradykinin with different separation voltages. MCE experimental conditions: capillary, coated silica capillary, $75 \mu \mathrm{m}$ i.d., $60 \mathrm{~cm}$ total length $(50 \mathrm{~cm}$ to the detector); phosphate buffer ( $20 \mathrm{mM}, \mathrm{pH}$ 7); separation voltage 5, 10 and $20 \mathrm{kV}$; driving pressure 50 mbar; hydrodynamic injection $50 \mathrm{mbar}$ for $5 \mathrm{~s}$; temperature $25^{\circ} \mathrm{C}$. (b) Diffusion coefficients, (c) hydrodynamic radiuses and (d) effective charges of bradykinin with a separation voltage of 5,10 and $20 \mathrm{kV}$, respectively. TDA experimental results were also plotted in (c) for comparison.

experiments. Theoretically, the retention time of the phenol peak should remain the same in all experiments, because phenol molecules are not affected by electric fields. Since EOF was minimized in this study, voltage induced thermal heating is believed to be the predominant reason for this neutral marker retention time variation. At higher temperatures, the viscosity of the solvent decreases, which would result in an increased solvent flow rate. In turn, the results suggest that temperature and viscosity corrections are important in MCE experiments.

Eqn (7) was then applied to calculate the diffusion coefficients of bradykinin in these three experiments. As plotted in Fig. 2b, bradykinin has larger diffusion coefficients at elevated voltages, which agrees with the fact that bradykinin ions would have stronger dispersion at higher temperatures in a solvent with lower viscosity. ${ }^{69}$ After the temperature and viscosity correction, Fig. $2 c$ and d plot the hydrodynamic radius and effective charge of bradykinin, which were calculated using eqn (10) and (12), respectively. Although the diffusion coefficient of bradykinin varies under different experimental conditions, the hydrodynamic radius $\left(R_{\mathrm{h}}\right)$ and its effective charge number $(n)$ remain relatively unchanged. The calculated $R_{\mathrm{h}}$ and $n$ are $9.17 \pm$ $0.18 \AA$ and $2.16 \pm 0.03,9.13 \pm 0.04 \AA$ and $2.26 \pm 0.13$, and $9.17 \pm$ $0.17 \AA$ and $2.32 \pm 0.09$ with an applied separation voltage of 5 , 10 and $20 \mathrm{kV}$, respectively. Conventional TDA experiments were also carried out with the measured hydrodynamic radius of bradykinin plotted in Fig. 2c for comparison. An $R_{\mathrm{h}}$ of $9.23 \pm$ $0.05 \AA$ was obtained using the TDA method. The agreement between TDA and MCE results confirms the validity of this MCE based ion hydrodynamic radius measurement.

\section{Mixture analyses}

As one type of CE method, MCE could achieve mixture separation, which allows measuring the ion hydrodynamic radius and effective charge of each peak presented in the electropherogram. As proof-of-concept demonstrations, MCE analyses were performed for a two-peptide mixture and a two-protein mixture. Fig. 3a shows the separated electropherogram of bradykinin (0.24 $\mu \mathrm{mol} \mathrm{mL}{ }^{-1}$ ), VEALYL (pentapeptide, $0.71 \mu \mathrm{mol} \mathrm{mL}^{-1}$ ) and the neutral marker, phenol (1/4000, volume ratio). The phosphate buffer (20 mM, pH 7) was used as the running buffer. 50 mbar and $20 \mathrm{kV}$ were applied as the driving and separation forces, respectively. Using eqn (10) and (12) in the theory section, $R_{\mathrm{h}}$ and $n$ could be obtained as $9.10 \pm 0.03 \AA$ and $2.45 \pm 0.02$ for bradykinin, and $7.33 \pm 0.23 \AA$ and $0.84 \pm 0.02$ for VEALYL.

For the mixture of lysozyme $\left(0.36 \mu \mathrm{mol} \mathrm{mL}{ }^{-1}\right)$, BSA $(0.07$ $\left.\mu \mathrm{mol} \mathrm{mL}{ }^{-1}\right)$ and phenol (1/500, volume ratio), the same running buffer was used as in the previous case (phosphate buffer, $20 \mathrm{mM}, \mathrm{pH} 7$ ), and $30 \mathrm{mbar}$ and $10 \mathrm{kV}$ were applied to drive and separate those molecules. As shown in Fig. 3b, the lysozyme peak appears before phenol, while the BSA peak appears after phenol. Since a positive voltage is applied and the electric field drives cations towards the UV detector, lysozyme is positively charged, while BSA is negatively charged under the experimental solvent conditions. After performing the peak fitting and ion retention time calculations (eqn (12)), it was found that lysozyme and BSA have an effective charge of $0.91 \pm 0.04$ and $-4.38 \pm 0.02$, respectively. The calculated hydrodynamic radiuses of lysozyme and BSA are 19.01 $\pm 0.74 \AA$ and $38.26 \pm 0.86 \AA$, which agrees well with those acquired using TDA methods from the literature. ${ }^{78}$

\section{Coupling with MD simulations for protein structure analyses}

As depicted in Fig. 1, the hydrodynamic radius obtained from MCE experiments could be further utilized as a selection rule
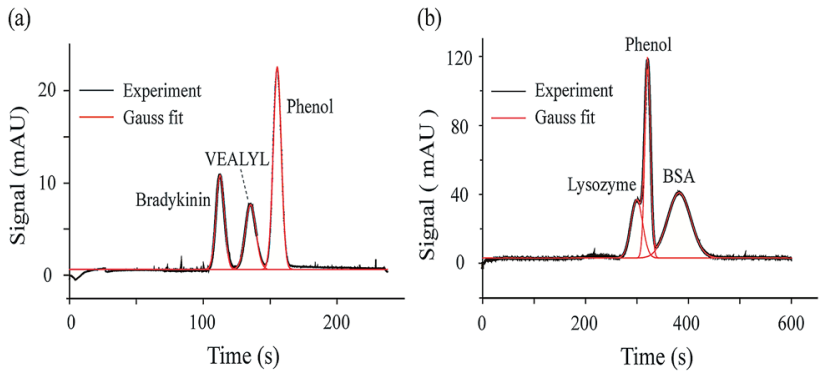

Fig. 3 Mixture analyses. (a) The mixture of VEALYL and bradykinin with phenol as the neutral marker. $50 \mathrm{mbar}$ and $20 \mathrm{kV}$ were applied during the separation process. (b) The mixture of BSA and lysozyme with phenol as the neutral marker. $30 \mathrm{mbar}$ and $10 \mathrm{kV}$ were applied during the separation process. Other MCE experimental conditions: capillary, coated silica capillary, $75 \mu \mathrm{m}$ i.d., $60 \mathrm{~cm}$ total length $(50 \mathrm{~cm}$ to the detector); phosphate buffer ( $20 \mathrm{mM}, \mathrm{pH}$ 7); hydrodynamic injection 50 mbar for $5 \mathrm{~s}$; temperature $25^{\circ} \mathrm{C}$. 
for MD simulations, so that the most probable protein conformation under experimental solvent conditions could be obtained. ${ }^{32,33}$ Briefly, a lot of possible conformations of a protein could be acquired from the MD simulation, and a selection rule is needed to pick out the most probable protein conformation. By assuming that this protein has a spheroidal shape and applying the Stokes flow equation at low Reynolds numbers, the ion hydrodynamic radius of this protein obtained from the MCE experiment defines a mathematical relationship between the spheroid length to diameter ratio $(l / d)$ and its radius $(c$ and $d=$ $2 c$ ). This mathematical relationship (named as the shape restrain curve as shown in Fig. 4a) could then be used as the selection rule to find the most probable protein conformation from MD simulation results.

As described earlier, the current method is applicable over a wide range of salt concentrations and $\mathrm{pHs}$, which could cover the biological environments of most proteins. First, MCE experiments were performed for three proteins: insulin, cytochrome C and RNase A, respectively. Cytochrome C $(2.26 \mu \mathrm{mol}$ $\left.\mathrm{mL}^{-1}\right)$ was prepared in the sodium chloride solution $(22 \mathrm{mmol}$

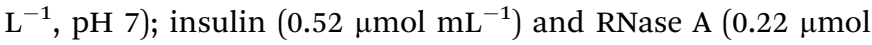
$\mathrm{mL}^{-1}$ ) were prepared in the disodium hydrogen-phosphate citric acid buffers ( $10 \mathrm{mM}, \mathrm{pH} 2.2) .50 \mathrm{mbar}$ and $10 \mathrm{kV}$ were applied as the driving and separation forces with the addition of $1 / 1000$ (volume ratio) phenol as the neutral marker. The hydrodynamic radiuses measured from MCE experiments of these three proteins are shown in Fig. 4a insets, and the effective charge of insulin, cytochrome $\mathrm{C}$ and RNase A was $3.92 \pm$ $0.06,3.91 \pm 0.53$ and $4.76 \pm 0.09$, respectively. MD simulations were also performed in parallel. The spheroid approximation
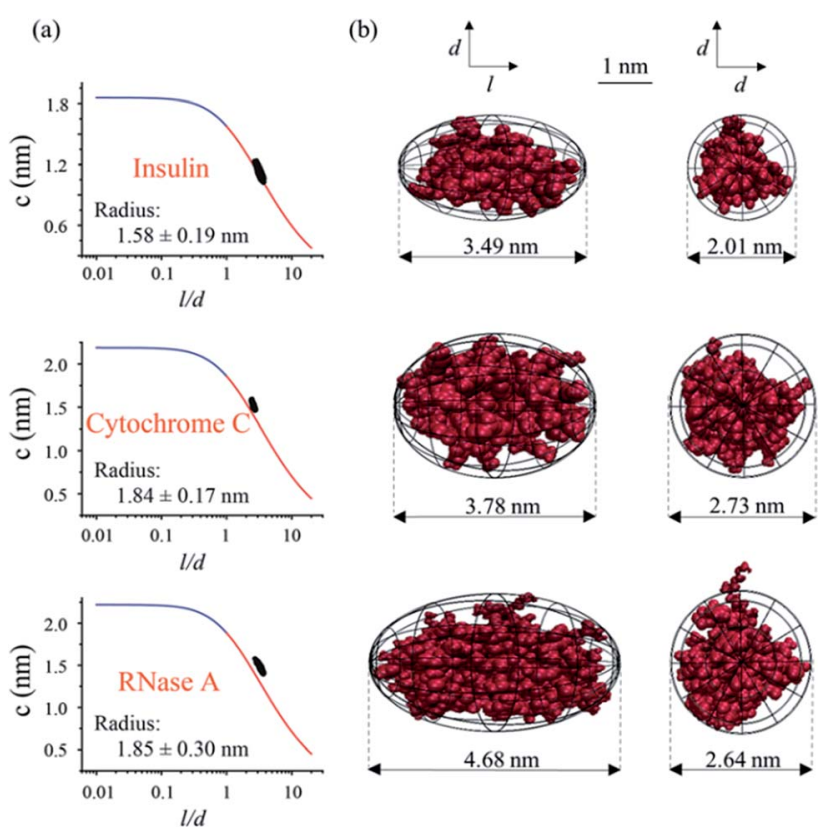

Fig. 4 The coupling of MCE results with MD simulations for protein conformation analyses. (a) The shape restrain curves obtained from MCE experiments were used to match MD simulations. (b) Side and top views of the most probable protein conformations. Note: $l$ and $d$ are the length and diameter of the spheroid, and $d=2 c$. was applied on both MCE results and simulation results as shown in Fig. 1. Fig. 4a plots the shape restrain curves obtained from MCE experiments, and 10000 simulated possible conformations for each protein (after spheroid approximation) were also plotted as black dots in these figures. The most probable protein conformation was selected if its corresponding dot has the closest distance from the shape restrain curve. Details about this spheroid approximation and matching processes could also be found in the literature. ${ }^{32,33,79}$ As shown in Fig. 4b, we described the conformations of these three proteins from side and top views respectively, and all of these three proteins have spheroid shapes but with different $l$ and $d$ values. The most probable conformations of these three proteins obtained using this MCE based method agree well with those from PDB.

\section{Protein charge and conformation variations at different $\mathbf{p H s}$}

As one of the most important bio-environment parameters, $\mathrm{pH}$ has large effects on both charge states and conformations of proteins. As a result, proteins would show very distinct characteristics under different $\mathrm{pH}$ conditions, such as solubility and bio-activity. In reality, different proteins may have distinct tolerances to $\mathrm{pH}$ changes in solution. As a demonstration, the conformations and charge states of two proteins, BSA and lysozyme, were analyzed and compared under different $\mathrm{pH}$ conditions. BSA and lysozyme were diluted in the disodium hydrogen-phosphate citric acid buffers at different $\mathrm{pH}$ values. Four different $\mathrm{pH}$ conditions were tested for both BSA and lysozyme, and the final concentrations of BSA and lysozyme were $0.05 \mu \mathrm{mol} \mathrm{mL}{ }^{-1}$ and $0.14 \mu \mathrm{mol} \mathrm{mL}^{-1}$, respectively, for all cases. After performing MCE experiments, it was found that the hydrodynamic radius and charge state of BSA generally increase at lower pHs (Fig. 5a and b). Its radius increases from 37.84 to $52.51 \AA$, while its charge state varies from -3.93 to 15.45 . The effective charge of BSA would change from negative to positive at a $\mathrm{pH}$ of $\sim 4.4$, which agrees well with the isoelectric point (pI) value of BSA in the literature. ${ }^{80,81}$ By coupling with MD simulation, the most probable conformations of BSA under different $\mathrm{pH}$ conditions were also obtained and are shown in Fig. 5c. Under pH neutral or 6.0 conditions, BSA exhibits the "basic" conformation. ${ }^{82}$ An unfolding process of BSA under lower $\mathrm{pH}$ conditions could be well observed. First BSA is a "Y" shaped "dish" like protein at $\mathrm{pH} 6.0$ and 7.0, and its two branches would spread further from each other at lower pHs. With decreased $\mathrm{pH}$, the net charge of BSA increases dramatically from $\sim-3.93$ to $\sim+\mathbf{1 5 . 4 5}$. The increased number of charged residues would induce intense electrostatic forces within a BSA molecule, causing the collapse and/or unfolding of the molecule. ${ }^{83}$ As the solvent $\mathrm{pH}$ decreases to 3.4 , it is found that this "Y" shaped "dish" like BSA would transform into a "V" shaped "rugby" like spheroid. Disulfide bonds and hydrophobic interactions within BSA would break together with reduced helical content, which causes BSA unfolding into the "F" conformation. ${ }^{82}$

Lysozyme is an important immune related enzyme present in many body secretions, and it is known to be thermally stable. The crystal structure of lysozyme was clarified by Phillips et al. 
(a)

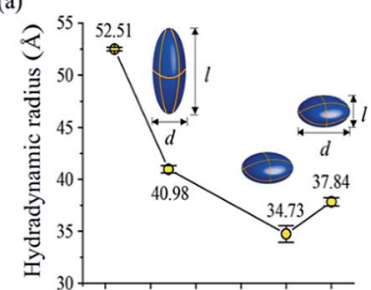

(b)

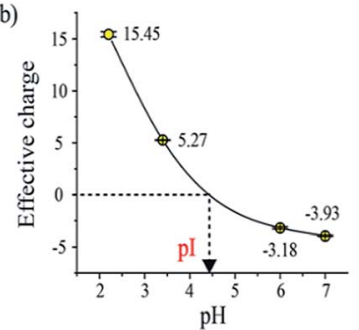

(c)

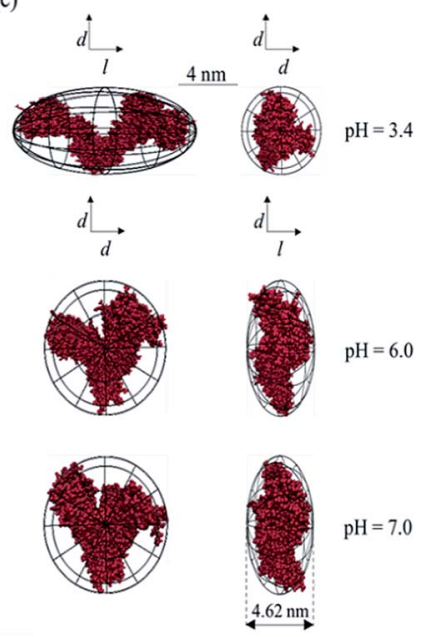

Fig. 5 Conformation and charge state variations of BSA under different $\mathrm{pH}$ conditions. (a) Hydrodynamic radiuses and (b) effective charges of BSA at different $\mathrm{pHs}$, and pl indicates the isoelectric point of BSA. (c) Side and top views of BSA conformations under different $\mathrm{pH}$ conditions.

through X-ray analysis, ${ }^{84}$ and it has a spheroid shape. As shown in Fig. 6, the hydrodynamic radius of lysozyme remains relatively stable over the whole measured $\mathrm{pH}$ range (Fig. 6a). Although the effective charge of lysozyme also increases, the absolute increment is much smaller (from +0.82 to +4.99 , Fig. 6b) when compared to that of BSA. Protein conformation prediction results show that lysozyme is a "rugby" shaped protein, and its conformation only has very small variations in terms of $\mathrm{pH}$ changes. With reduced effective charge variation, less intense electrostatic forces exist within lysozyme, and the 4 disulfide bonds within lysozyme are retained, which in turn helps in maintaining its stereo-structure at different pHs. The conformations of BSA and lysozyme under different $\mathrm{pH}$ conditions match well with those in the literature, ${ }^{82,85}$ suggesting that

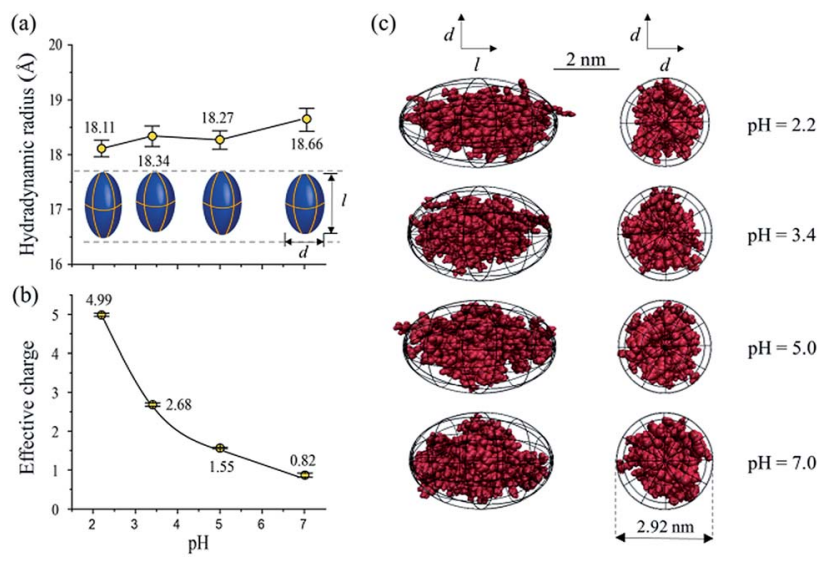

Fig. 6 Conformation and charge state variations of lysozyme under different $\mathrm{pH}$ conditions. (a) Hydrodynamic radiuses and (b) effective charges of lysozyme at different pHs. (c) Side and top views of lysozyme conformations under different $\mathrm{pH}$ conditions.

this MCE based method is a reliable method in terms of protein structure analysis under different solvent conditions.

\section{Conclusions}

A new MCE based method was proposed in this work to measure the hydrodynamic radiuses and effective charges of proteins under different solvent conditions. Without the need for chemical modifications, proteins in mixtures could be separated and analyzed in a single experiment. Using MCE experimental results as a selection rule for MD simulations, the most probable protein conformations could be acquired. This method has been demonstrated to analyze peptide and protein mixtures. The structures and charges of three proteins were analyzed under conditions close to their native $\mathrm{pH}$. The effects of $\mathrm{pH}$ changes on protein conformation transitions and charge state variations were also explored for BSA and lysozyme. BSA and lysozyme react significantly differently to $\mathrm{pH}$ changes. Besides the large charge state variations, BSA shows a significant conformation variation from a "Y" shaped "dish" like protein to a "V" shaped rugby like protein, while lysozyme maintains a relatively stable rugby like shape. By using pressure to drive the liquid flow and minimizing the EOF in separation capillaries, an ordinary CE instrument could be used to perform these MCE based experiments. Furthermore, besides proteins, radiuses and net charges of other molecules, such as drugs, glycans, lipids and even nanoparticles ${ }^{86,87}$ could also be analyzed using this method. Compared to conventional structural biology technologies, low-resolution protein shape information is mainly obtained from MCE experiments, and MD simulations are required for protein conformation predictions. However, as a liquid-phase analogue of IMS, this MCE based method is rapid in terms of speed and has low sample consumption rates (nanoliter level), which makes it potentially applicable in top-down proteomics for large-scale protein structure and function investigations. For instance, a small fraction of a biological sample could be subjected to this MCEbased analysis first to rapidly pick out structurally abnormal proteins. The rest of the sample could then be subjected to a conventional structural biology analysis process to resolve the high-resolution structures of abnormal proteins identified in the previous MCE experiments.

\section{Conflicts of interest}

There are no conflicts to declare.

\section{Acknowledgements}

This work was supported by the NNSFC (201827810, 61635003), and National Key Research and Development Plan (2018YFF0212500).

\section{References}

1 L. Jia, L. Zhang, C. Shao, E. Song, W. Sun, M. Li and Y. Gao, PLoS One, 2009, 4, e5146. 
2 R. S. Lee, L. Zhang, A. Berger, M. G. Lawrence, J. Song, B. Niranjan, R. G. Davies, N. L. Lister, S. K. Sandhu, M. A. Rubin, G. P. Risbridger, R. A. Taylor, D. S. Rickman, L. G. Horvath and R. J. Daly, Neoplasia, 2019, 21, 389-400.

$3 \mathrm{~W}$. Bode, P. Schwager and R. Huber, Proteolysis and Physiological Regulation, 1976, vol. 11, pp. 43-76.

4 J. D. Watson, R. A. Laskowski and J. M. Thornton, Curr. Opin. Struct. Biol., 2005, 15, 275-284.

5 B. Over, P. Matsson, C. Tyrchan, P. Artursson, B. C. Doak, M. A. Foley, C. Hilgendorf, S. E. Johnston, M. D. Lee IV and R. J. Lewis, Nat. Chem. Biol., 2016, 12, 1065.

6 I. Gitlin, J. D. Carbeck and G. M. Whitesides, Angew. Chem., Int. Ed., 2006, 45, 3022-3060.

7 C. T. Zahler and B. F. Shaw, Chem.-Eur. J., 2019, 25, 75817590.

8 G. Pearson, F. Robinson, T. Beers Gibson, B.-e. Xu, M. Karandikar, K. Berman and M. H. Cobb, Endocr. Rev., 2001, 22, 153-183.

9 M. D. Brand and T. C. Esteves, Cell Metab., 2005, 2, 85-93.

10 M. Sunde, L. C. Serpell, M. Bartlam, P. E. Fraser, M. B. Pepys and C. C. Blake, J. Mol. Biol., 1997, 273, 729-739.

11 D. S. Wishart, B. D. Sykes and F. M. Richards, J. Mol. Biol., 1991, 222, 311-333.

12 H. E. Huxley, J. Mol. Biol., 1963, 7, 281-308.

13 C.-Y. Huang, V. Olieric, P. Ma, N. Howe, L. Vogeley, X. Liu, R. Warshamanage, T. Weinert, E. Panepucci and B. Kobilka, Acta Crystallogr., Sect. D: Struct. Biol., 2016, 72, 93-112.

14 C. L. Moralesperez, C. M. Noviello and R. E. Hibbs, Nature, 2016, 538, 411-415.

15 R. Ramautar, Adv. Clin. Chem., 2016, 74, 1-34.

16 R. Gahoual, E. Leizewagner, P. Houze and Y. Francois, Rapid Commun. Mass Spectrom., 2018, 33, 11-19.

17 M. Dams, J. L. Doressousa, R. Lamers, A. Treumann and S. Eeltink, Chromatographia, 2019, 82, 101-110.

18 K. L. Ford, W. Zeng, J. L. Heazlewood and A. Bacic, Frontiers in Plant Science, 2015, 6, 674.

19 A. Leitner, Chem. Sci., 2016, 7, 4792-4803.

20 B. Schindler, A. D. Depland, G. Renoispredelus, G. Karras, B. Concina, G. Celep, J. Maurelli, V. Loriot, E. Constant and R. Bredy, Int. J. Ion Mobility Spectrom., 2017, 20, 119-124.

21 S. M. Stow, T. J. Causon, X. Zheng, R. T. Kurulugama, T. Mairinger, J. C. May, E. E. Rennie, E. S. Baker, R. D. Smith and J. A. Mclean, Anal. Chem., 2017, 89, 90489055.

22 T. Jiang, M. He, D. Guo, Y. Zhai and W. Xu, Phys. Chem. Chem. Phys., 2016, 18, 12058-12064.

23 C. Klein, S. M. Cologna, R. T. Kurulugama, P. S. Blank, E. Darland, A. Mordehai, P. S. Backlund and A. L. Yergey, Analyst, 2018, 143, 4147-4154.

24 T. Jiang, Y. Chen, L. Mao, A. G. Marshall and W. Xu, Phys. Chem. Chem. Phys., 2016, 18, 713-717.

25 H. Yang, S. Yang, J. Kong, A. Dong and S. Yu, Nat. Protoc., 2015, 10, 382.

26 S. Prasad, I. Mandal, S. Singh, A. Paul, B. Mandal, R. Venkatramani and R. Swaminathan, Chem. Sci., 2017, 8, 5416-5433.
27 H. Cottet, M. Martin, A. Papillaud, E. Souaid, H. Collet and

A. Commeyras, Biomacromolecules, 2007, 8, 3235-3243.

28 A. Alizadeh, C. A. N. De Castro and W. A. Wakeham, Int. J. Thermophys., 1980, 1, 243-284.

29 U. Tallarek, E. Rapp, T. W. J. Scheenen, B. Ernst and H. Van As, Anal. Chem., 2000, 72, 2292-2301.

30 H. Cottet, J. Biron and M. Martin, Anal. Chem., 2007, 79, 9066-9073.

31 B. A. Williams and G. Vigh, Anal. Chem., 1997, 69, 4410-4418. 32 M. He, P. Luo, J. Hong, X. Wang, H. Wu, R. Zhang, F. Qu, Y. Xiang and W. Xu, ACS Omega, 2019, 4, 2377-2386.

33 R. Zhang, H. Wu, M. He, W. Zhang and W. Xu, J. Phys. Chem. B, 2019, 123, 2335-2341.

34 R. Johann and P. Renaud, Electrophoresis, 2004, 25, 37203729.

35 A. Kamholz and P. Yager, Biophys. J., 2001, 80, 155-160.

36 M. A. Hayes, I. Kheterpal and A. G. Ewing, Anal. Chem., 1993, 65, 2010-2013.

37 J. E. Melanson, N. E. Baryla and C. A. Lucy, Trends Anal. Chem., 2001, 20, 365-374.

38 W. Zhang, M. He, T. Yuan and W. Xu, Electrophoresis, 2017, 38, 3130-3135.

39 Z. Hall and C. V. Robinson, J. Am. Soc. Mass Spectrom., 2012, 23, 1161-1168.

40 Y. Shi, M. J. Acerson, A. Abdolvahabi, R. A. Mowery and B. F. Shaw, J. Am. Chem. Soc., 2016, 138, 5351.

41 S. Rasouli, A. Abdolvahabi, C. M. Croom, D. L. Plewman, Y. Shi, J. I. Ayers and B. F. Shaw, J. Biol. Chem., 2017, 292, 19366-19380.

42 G. Irina, J. D. Carbeck and G. M. Whitesides, Angew. Chem., 2010, 45, 3022-3060.

43 E. Alexov and M. Gunner, Biophys. J., 1997, 72, 2075-2093.

44 J. Gao, F. A. Gomez, R. Härter and G. M. Whitesides, Proc. Natl. Acad. Sci. U. S. A., 1994, 91, 12027-12030.

45 A. Ibrahim, D. Koval, V. Kašička, C. Faye and H. Cottet, Macromolecules, 2013, 46, 533-540.

46 J. Chamieh, D. Koval, A. Besson, V. Kašička and H. Cottet, J. Chromatogr. A, 2014, 1370, 255-262.

47 T. Tůmová, L. Monincová, O. Nešuta, V. Čeřovský and V. Kašička, Electrophoresis, 2017, 38, 2018-2024.

48 M. B. Cammarata and J. S. Brodbelt, Chem. Sci., 2015, 6, 1324-1333.

49 N. A. Yewdall, T. M. Allison, F. G. Pearce, C. V. Robinson and J. A. Gerrard, Chem. Sci., 2018, 9, 6099-6106.

50 W. Chen, S. Li, X. Li, C. Zhang, X. Hu, F. Zhu, G. Shen and F. Feng, Chem. Sci., 2019, 10, 2179-2185.

51 H. Cottet, J.-P. Biron and M. Martin, Anal. Chem., 2007, 79, 9066-9073.

52 T. Le Saux and H. Cottet, Anal. Chem., 2008, 80, 1829-1832. 53 C. T. Culbertson and J. W. Jorgenson, Anal. Chem., 1994, 66, 955-962.

54 R. Datta and V. R. Kotamarthi, AIChE J., 1990, 36, 916-926. 55 R. Datta, Biotechnol. Prog., 2010, 6, 485-493.

56 A. Ibrahim, H. Ohshima, S. A. Allison and H. Cottet, J. Chromatogr. A, 2012, 1247, 154-164.

57 J. Ostergaard and H. Jensen, Anal. Chem., 2009, 81, 86448648. 
58 I. Frankel and H. Brenner, J. Fluid Mech., 1989, 204, 97-119. 59 R. Aris, Process Systems Engineering, 1999, vol. 1, pp. 109-120. 60 S. Upma, N. J. Gleason and J. D. Carbeck, Anal. Chem., 2005, 77, 806-813.

61 M. Kahlen, A. Engel and C. Van den Broeck, Phys. Rev. E, 2017, 95, 012144.

62 C. Willingham, V. Sedlak, F. Rossini and J. Westhaver, Ind. Eng. Chem., 1947, 39, 706-712.

63 J. W. Westhaver, J. Res. Natl. Bur. Stand., 1947, 38, 169-183. 64 G. I. Taylor, Proc. Roy. Soc. Lond. Math. Phys. Sci., 1953, 219, 186-203.

65 R. Aris, Proc. Roy. Soc. Lond. Math. Phys. Sci., 1956, 235, 6777.

66 P. Gebauer and P. BočEk, Anal. Chem., 1998, 69, 1557-1563. 67 F. E. P. Mikkers, F. M. Everaerts and T. P. E. M. Verheggen, J. Chromatogr. A, 1979, 169, 1-10.

68 Y. G. Tang, C. Yang, Q. H. Gong, C. J. Chai and C. Y. Lam, Anal. Chim. Acta, 2006, 561, 138-149.

69 J. Vlassakis and A. E. Herr, Anal. Chem., 2017, 89, 1278712796.

70 D. Dutta, Electrophoresis, 2018, 39, 760-769.

71 Z. Min, Y. L. Han, Q. I. Li and C. Yi, Chin. Sci. Bull., 2007, 52, 3325-3332.

72 N. Schmid, A. P. Eichenberger, A. Choutko, S. Riniker, M. Winger, A. E. Mark and W. F. van Gunsteren, Eur. Biophys. J., 2011, 40, 843.

73 M. J. Abraham, T. Murtola, R. Schulz, S. Páll, J. C. Smith, B. Hess and E. Lindahl, SoftwareX, 2015, 1, 19-25.
74 M. Rostkowski, M. H. M. Olsson, C. R. Sondergaard and J. H. Jensen, BMC Struct. Biol., 2011, 11, 6.

75 H. Li, A. D. Robertson and J. H. Jensen, Proteins, 2005, 61, 704-721.

76 T. A. Soares, X. Daura, C. Oostenbrink, L. J. Smith and W. F. Van Gunsteren, J. Biomol. NMR, 2004, 30, 407-422.

77 U. Essmann, L. Perera, M. L. Berkowitz, T. Darden, H. Lee and L. G. Pedersen, J. Chem. Phys., 1995, 103, 8577-8593.

78 W. L. Hulse and R. T. Forbes, Int. J. Pharm., 2011, 411, 64-68.

79 C. N. Davies, J. Aerosol Sci., 1975, 6, 273.

80 U. Łapińska, K. L. Saar, E. V. Yates, T. W. Herling, T. Müller, P. K. Challa, C. M. Dobson and T. P. Knowles, Phys. Chem. Chem. Phys., 2017, 19, 23060-23067.

81 Z. Peng, K. Hidajat and M. Uddin, J. Colloid Interface Sci., 2004, 271, 277-283.

82 J. F. Foster, Albumin Structure Function \& Uses, 1977, pp. 5384.

83 M. T. Donor, A. M. Mroz and J. S. Prell, Chem. Sci., 2019, 10, 4097-4106.

84 C. Blake, D. Koenig, G. Mair, A. North, D. Phillips and V. Sarma, Nature, 1965, 206, 757.

85 Y. Yamagata, M. Kubota, Y. Sumikawa, J. Funahashi, K. Takano, S. Fujii and K. Yutani, Biochemistry, 1998, 37, 9355-9362.

86 W. Ma, H. Ma, J.-F. Chen, Y.-Y. Peng, Z.-Y. Yang, H.-F. Wang, Y.-L. Ying, H. Tian and Y.-T. Long, Chem. Sci., 2017, 8, 18541861.

87 C. Jing, Z. Gu, T. Xie and Y.-T. Long, Chem. Sci., 2016, 7, 5347-5351. 